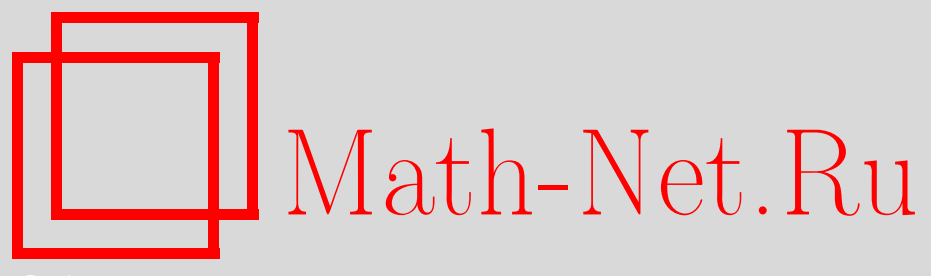

Е.-М. Ильгенфриц, С. А. Пастон, Г.-Ю. Пирнер, Е. В. Прохватилов, В. А. Франке, Квантовые поля на световом фронте, формулировка в координатах, близких к световому фронту, решеточное приближение, ТМФ, 2006, том 148, номер 1, 89-101

DOI: https://doi.org/10.4213/tmf2060

Использование Общероссийского математического портала Math-Net.Ru подразумевает, что вы прочитали и согласны с пользовательским соглашением http://www.mathnet.ru/rus/agreement

Параметры загрузки:

IP : 52.90 .164 .192

26 апреля 2023 г., 15:20:42

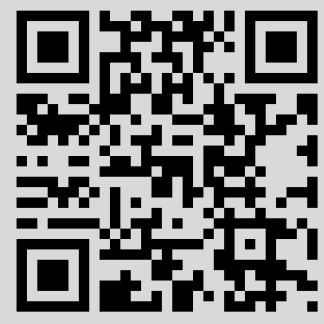




\title{
КВАНТОВЫЕ ПОЛЯ НА СВЕТОВОМ ФРОНТЕ, ФОРМУЛИРОВКА В КООРДИНАТАХ, БЛИЗКИХ К СВЕТОВОМУ ФРОНТУ, РЕШЕТОЧНОЕ ПРИБЛИЖЕНИЕ
}

\begin{abstract}
Приведен обзор идей, лежащих в основе квантования теории поля на световом фронте, в том числе, гамильтонов подход к проблеме связанных состояний на световом фронте, а также предельный переход к теории на световом фронте от формулировок теории в лоренцевых координатах (при квантовании на пространственноподобных гиперплоскостях), который имеет целью обеспечить эквивалентность этих вариантов теории. Описаны попытки найти форму такого предельного перехода для калибровочных теорий на решетке Вильсона.
\end{abstract}

Ключевые слова: калибровочные поля, световой фронт, гамильтонов подход, решетка.

\section{1. ВВЕДЕНИЕ}

Идея квантования релятивистских полей на световом фронте (СФ) была выдвинута Дираком [1], который ввел вместо лоренцевых координат $x^{0}, x^{1}, x^{2}, x^{3}$ координаты светового фронта

$$
x^{ \pm}=\left(x^{0} \pm x^{3}\right) / \sqrt{2}, \quad x^{\perp} \equiv\left(x^{1}, x^{2}\right) .
$$

При этом $x^{+}$играет роль времени, а $x^{-}$("светоподобная" координата) - роль одной из пространственных координат, $x^{k}$ - "поперечные" координаты, $k=1,2$.

Теория поля квантуется на гиперплоскости $x^{+}=0$, которая касается светового конуса и поэтому соответствует световому фронту. Роль гамильтониана играет генератор трансляций вдоль $x^{+}$, т.е. оператор $P_{+}=\left(P_{0}+P_{3}\right) / \sqrt{2}$, а оператор

Статья основана на лекции, прочитанной на школе теоретической физики им. В. А. Фока.

${ }^{*}$ Institut fur Physik, Humboldt-Universitaet zu Berlin, Germany.

E-mail: ilgenfri@physik.hu-berlin.de

${ }^{\dagger}$ Санкт-Петербургский государственный университет, Санкт-Петербург, Россия. E-mail: paston@pobox.spbu.ru, Evgeni.Prokhvat@pobox.spbu.ru, franke@pobox.spbu.ru

${ }^{\ddagger}$ Institute for Theoretical Physics, University of Heidelberg, Germany. E-mail: pir@tphys.uni-heidelberg.de 
$P_{-}=\left(P_{0}-P_{3}\right) / \sqrt{2}$, отвечающий трансляциям вдоль $x^{-}$, играет роль одной из компонент импульса ("светоподобной" компоненты).

Одно из преимуществ квантования на СФ - это формальное упрощение проблемы описания квантового вакуумного состояния в теории поля. Обычно в лоренцевых координатах поля́ квантуются при фиксированном времени, например, при $x^{0}=0$, и их фурье-образы просто связаны с “голыми" операторами рождения и уничтожения $a^{+}(p)$ и $a(p)$. "Голый" (или "математический") вакуум определяется условием

$$
a(p)|0\rangle=0 .
$$

Такое состояние соответствует вакууму свободной теории и не совпадает с физическим вакуумом теории со взаимодействием. При решении стационарного уравнения Шредингера в фоковском пространстве над этим математическим вакуумом требуется описать физическое вакуумное состояние в терминах "голого" вакуума и "голых" операторов рождения. Такое описание в принципе возможно, если введены ультрафиолетовая и инфракрасная регуляризации. Однако это описание чрезвычайно сложно (в простейших моделях часто ограничиваются "гауссовым" приближением [2]).

При квантовании в координатах СФ существенно, что светоподобная компонента импульса $P_{-}$неотрицательна, причем для состояний с положительным квадратом массы $P_{-}>0$. Поэтому при отсутствии безмассовых физических частиц состояние с импульсом $p_{-}=0$ формально описывает физический вакуум, т.е. отвечает также и минимуму оператора $P_{+}$. Вводя "голые" операторы рождения и уничтожения на СФ, можно определить с их помощью соответствующий математический вакуум. В силу структуры оператора импульса $P_{-}$математический вакуум отвечает минимуму этого оператора, т.е. совпадает с физическим вакуумом (пока формально из-за присутствия расходимостей в теории). Ниже это будет продемонстрировано на примере теории скалярного поля.

Фоковское пространство, построенное над указанным математическим вакуумом на СФ, может быть использовано для описания решений соответствующего аналога стационарного уравнения Шредингера, определяющего массы $M$ связанных состояний при фиксированных значениях импульсов $P_{-}$и $P_{\perp} \equiv\left(P_{1}, P_{2}\right)$ :

$$
\begin{gathered}
P_{+}|\psi\rangle=H|\psi\rangle=p_{+}|\psi\rangle, \\
P_{-}|\psi\rangle=p_{-}|\psi\rangle, \quad P_{\perp}|\psi\rangle=0 .
\end{gathered}
$$

При этом $M^{2}=2 p_{+} p_{-}$.

Рассмотрим в качестве примера теорию скалярного поля (с массой $m)$, задаваемую лагранжевой плотностью $L(x)$ :

$L=\frac{1}{2} \partial_{\mu} \varphi \partial^{\mu} \varphi-\frac{1}{2} m^{2} \varphi^{2}-U(\varphi)=\partial_{+} \varphi \partial_{-} \varphi-\frac{1}{2} \partial_{k} \varphi \partial_{k} \varphi-\frac{1}{2} m^{2} \varphi^{2}-U(\varphi), \quad \partial_{\mu} \varphi \equiv \frac{\partial \varphi}{\partial x^{\mu}}$.

Для построения канонического формализма при $x^{+}=0$ введем фурье-преобразование поля $\varphi(x)$ по координате $x^{-}$, учитывая неотрицательность импульса $p_{-}$:

$$
\varphi\left(x^{-}, x^{\perp}\right)=\frac{1}{\sqrt{2 \pi}} \int_{0}^{\infty} d p_{-}\left(2 p_{-}\right)^{-1 / 2}\left(a^{+}\left(p_{-}, x^{\perp}\right) e^{i p_{-} x^{-}}+a\left(p_{-}, x^{\perp}\right) e^{-i p_{-} x^{-}}\right) .
$$


Тогда

$$
\int d x^{-} \partial_{+} \varphi \partial_{-} \varphi=\int_{0}^{\infty} d p_{-} \frac{1}{2 i}\left(\partial_{+} a^{+}\left(p_{-}, x^{\perp}\right) a\left(p_{-}, x^{\perp}\right)-a^{+}\left(p_{-}, x^{\perp}\right) \partial_{+} a\left(p_{-}, x^{\perp}\right)\right),
$$

что представляет собой каноническую форму, в которой $a\left(p_{-}, x^{\perp}\right)$ и $i a^{+}\left(p_{-}, x^{\perp}\right)$ играют роль канонически сопряженных переменных. Квантовые операторы $a\left(p_{-}, x^{\perp}\right)$ и $a^{+}\left(p_{-}, x^{\perp}\right)$ удовлетворяют (при $\left.x^{+}=0\right)$ перестановочным соотношениям

$$
\begin{aligned}
{\left[a\left(p_{-}, x^{\perp}\right), a^{+}\left(q_{-}, y^{\perp}\right)\right] } & =\delta\left(p_{-}-q_{-}\right) \delta^{2}\left(x^{\perp}-y^{\perp}\right), \\
{\left[a\left(p_{-}, x^{\perp}\right), a\left(q_{-}, y^{\perp}\right)\right] } & =\left[a^{+}\left(p_{-}, x^{\perp}\right), a^{+}\left(q_{-}, y^{\perp}\right)\right]=0 .
\end{aligned}
$$

Используя выражение для тензора энергии-импульса,

$$
T_{\mu \nu}=\partial_{\mu} \varphi \partial_{\nu} \varphi-g_{\mu \nu} L
$$

можно найти оператор $P_{-}$:

$$
\begin{aligned}
P_{-} & =\int d^{2} x^{\perp} \int d x^{-} T_{--}=\int d^{2} x^{\perp} \int d x^{-}\left(\partial_{-} \varphi\right)^{2}= \\
& =\int d^{2} x^{\perp} \int_{0}^{\infty} d p_{-} p_{-} a^{+}\left(p_{-}, x^{\perp}\right) a\left(p_{-}, x^{\perp}\right),
\end{aligned}
$$

где отброшена бесконечная постоянная. Определим состояние математического вакуума $|0\rangle$ условиями

$$
a\left(p_{-}, x^{\perp}\right)|0\rangle=0, \quad p_{-} \geqslant 0 .
$$

Тогда $P_{-}|0\rangle=0$, и состояние $|0\rangle$ можно рассматривать как физический вакуум. Чтобы это имело место, и пришлось отбросить бесконечную постоянную в равенстве (9).

Гамильтониан можно получить стандартным способом из лагранжиана, записанного в терминах переменных $a\left(p_{-}, x^{\perp}\right)$ и $a^{+}\left(p_{-}, x^{\perp}\right)$. В рассматриваемой теории он совпадает (с точностью до постоянной) с выражением

$$
P_{+}=\int d^{2} x^{\perp} \int d x^{-} T_{-+}=\int d^{2} x^{\perp} \int d x^{-}\left(\frac{1}{2} \partial_{k} \varphi \partial_{k} \varphi+\frac{1}{2} m^{2} \varphi^{2}+U(\varphi)\right) .
$$

Сформулируем кратко трудности квантования на СФ

1. Ультрафиолетовые расходимости в квантовой теории поля требуют введения регуляризации и последующей перенормировки теории. При квантовании на СФ трудно ввести регуляризацию, сохраняющую лоренцеву и калибровочную симметрии. Неинвариантные регуляризации усложняют процедуру перенормировки, в частности, ведут к появлению необычных контрчленов и связанных с ними новых произвольных констант. При численном непертурбативном решении уравнения Шредингера на СФ необходимо работать с регуляризованной теорией и подбирать константы так, чтобы результаты вычислений слабо зависели от параметров регуляризации.

2. Квантование на СФ связано со специфическими особенностями и расходимостями при стремлении к нулю импульса $p_{-}$"голых" квантов. С точки зрения 
лоренцевых координат эти расходимости можно интерпретировать как ультрафиолетовые, так как предел $p_{-} \rightarrow 0$ достигается в области $p_{3} \rightarrow \infty$ при условии, что $p^{2}=m^{2}$. Однако если регуляризовать теорию путем обрезания импульса $p_{-}$ $\left(p_{-} \geqslant \varepsilon>0\right)$, то исключаются и вакуумные эффекты. Физический вакуум совпадает с "голым" вакуумом, при этом не может быть конденсатов и спонтанного нарушения симметрии вакуума. Все вакуумные эффекты должны быть учтены в виде дополнительных членов в гамильтониане на СФ Получить эти члены достаточно трудно. Например, их роль могут играть те контрчлены, которые перенормируют сингулярности при $p_{-} \rightarrow 0$ и восстанавливают результаты лоренц-ковариантной теории возмущений по константе взаимодействия во всех порядках [3], [4].

Можно вводить эти члены на основании полуфеноменологических соображений, например, изучая предельный переход на СФ, начиная с теории, квантованной на пространственноподобной поверхности, близкой к СФ (см. работы [5], [6]). Этот способ будет описан ниже. Предпринимались также попытки учесть вакуумные эффекты при квантовании калибровочных теорий на СФ, опираясь на анализ точного операторного решения модели Швингера [7].

3. При расчетах спектров масс связанных состояний приходится ограничиваться конечным числом степеней свободы для квантовых полей. С этой целью можно ввести, например, ограничение для координат и периодические граничные условия для полей по этим координатам, $\left|x^{-}\right| \leqslant L, x^{\perp^{2}} \leqslant L_{\perp}^{2}$, а также ограничить получаемый дискретный набор импульсов. Канонический формализм для такой формулировки содержит сложные связи, обусловленные присутствием нулевых фурье-мод полей по координате $x^{-}$. Для калибровочных полей на СФ эта проблема затрагивалась в работе [8].

Решая уравнение Шредингера в фоковском пространстве на СФ при фиксированном полном импульсе связанных состояний $\left(p_{-}=p_{n}=(\pi n) / L, p_{\perp}=0\right)$ и при условии $p_{n}>0$, а также при обрезании по поперечным импульсам отдельных возбуждений, мы получаем при каждом целом $n$ (и ограниченном наборе поперечных импульсов) конечномерные подпространства фоковского пространства, зависящие от $n$. Массы связанных состояний получаются как предел при $n \rightarrow \infty$ значений $m_{n}^{2}=2 p_{n,+} p_{n,-}$, найденных в каждом из подпространств. Для ряда рассмотренных $(1+1)$-мерных моделей значения $m_{n}^{2}$ практически перестают зависеть от $n$ уже при не слишком больших $n$ [9]. Однако для $(3+1)$-мерных теорий размерности фоковских подпространств зависят от всего набора импульсов $p_{\perp}$, при этом вычисления значительно усложняются [10]. Кроме того, указанная регуляризация нарушает лоренцеву (и калибровочную) симметрию, что усложняет процедуру перенормировки и восстановления симметрии в пределе снятия регуляризации.

Другой метод регуляризации - это ограничение числа "голых" квантов, участвующих в построении волновой функции связанного состояния в фоковском пространстве на СФ, т.е. "обрезание" фоковского пространства по числу “частиц" (метод Тамма-Данкова на СФ) [11]. При этом подходе можно не ограничивать координаты. Однако переход к фоковскому подпространству с увеличением числа "частиц" (т.е. голых квантов) сталкивается со значительными техническими трудностями. 


\section{2. МЕТОД ПРЕДЕЛЬНОГО ПЕРЕХОДА К ГАМИЛЬТОНИАНУ НА СФ}

Квантование теории поля на СФ можно трактовать как формальный предел квантования в лоренцевой системе отсчета, движущейся относительно искомых связанных состояний поля со скоростью, приближающейся к скорости света. Параметризуем соответствующее лоренцево преобразование координат $x \rightarrow x^{\prime}$ с помощью параметра $\eta>0, \quad \eta \rightarrow 0$ :

$$
x^{\prime+}=\frac{\sqrt{2}}{\eta} x^{+}, \quad x^{\prime-}=\frac{\eta}{\sqrt{2}} x^{-}, \quad x^{\perp}=x^{\perp},
$$

где $x^{\prime \pm}=\left(x^{\prime 0} \pm x^{\prime 3}\right) / \sqrt{2}$. Плоскость квантования полей $x^{\prime 0}=0$ аппроксимирует при $\eta \rightarrow 0$ плоскость СФ

Для дальнейшего обсуждения удобнее перейти от “быстродвижущейся" лоренцевой системы координат $x^{\prime}$ к координатам $\tilde{x}$ :

$$
\tilde{x}^{+}=\eta x^{\prime 0}=x^{+}+\frac{\eta^{2}}{2} x^{-}, \quad \tilde{x}^{-}=\eta^{-1}\left(x^{\prime 0}-x^{\prime 3}\right)=x^{-}, \quad \tilde{x}^{\perp}=x^{\perp},
$$

при этом плоскость $\tilde{x}^{+}=0$ совпадает с плоскостью $x^{\prime 0}=0$, и при $\eta \rightarrow 0$ координаты $\tilde{x}$ переходят в координаты СФ

Метрический тензор, отвечающий этим координатам, имеет ненулевые компоненты

$$
\begin{array}{lll}
\tilde{g}_{+-}(\tilde{x})=\tilde{g}_{-+}(\tilde{x})=1, & \tilde{g}_{--}(\tilde{x})=-\eta^{2}, & \tilde{g}_{k k}(\tilde{x})=-1, \\
\tilde{g}^{+-}(\tilde{x})=\tilde{g}^{-+}(\tilde{x})=1, & \tilde{g}^{++}(\tilde{x})=\eta^{2}, & \tilde{g}^{k k}(\tilde{x})=-1 .
\end{array}
$$

Продемонстрируем метод предельного перехода на примере теории скалярного поля в этих координатах. Плотность лагранжиана определим следующим образом:

$$
\begin{aligned}
L(\tilde{x}) & =\sqrt{\tilde{g}(\tilde{x})}\left(\frac{1}{2} \partial_{\mu} \varphi(\tilde{x}) \partial_{\nu} \varphi(\tilde{x}) \tilde{g}^{\mu \nu}(\tilde{x})-\frac{1}{2} m^{2} \varphi^{2}(\tilde{x})-\lambda \varphi^{4}(\tilde{x})\right)= \\
& =\tilde{\partial}_{+} \varphi(\tilde{x}) \tilde{\partial}_{-} \varphi(\tilde{x})+\frac{\eta^{2}}{2}\left(\tilde{\partial}_{+} \varphi(\tilde{x})\right)^{2}-\frac{1}{2}\left(\partial_{\perp} \varphi(\tilde{x})\right)^{2}-\frac{1}{2} m^{2} \varphi^{2}(\tilde{x})-\lambda \varphi^{4}(\tilde{x}),
\end{aligned}
$$

где $\lambda$ - константа взаимодействия.

Определим канонические переменные при $\tilde{x}^{+}=0$ как

$$
\varphi(\tilde{x}), \quad \Pi(\tilde{x})=\frac{\delta L}{\delta\left(\tilde{\partial}_{+} \varphi\right)}=\eta^{2} \tilde{\partial}_{+} \varphi+\tilde{\partial}_{-} \varphi .
$$

Гамильтониан принимает вид

$$
H(\eta)=\int d^{2} x^{\perp} \int d \tilde{x}^{-}\left(\frac{\left(\Pi-\tilde{\partial}_{-} \varphi\right)^{2}}{2 \eta^{2}}+\frac{1}{2}\left(\partial_{\perp} \varphi\right)^{2}+\frac{1}{2} m^{2} \varphi^{2}+\lambda \varphi^{4}\right) .
$$

Определим "голые" операторы рождения и уничтожения с помощью следующего фурье-преобразования:

$$
\begin{aligned}
& \varphi(\tilde{x})=(2 \pi)^{-3 / 2} \int d^{2} \tilde{p} d \tilde{p}_{-} \frac{1}{\sqrt{2 \omega_{p}}}\left[a(\tilde{p})+a^{+}(-\tilde{p})\right] e^{-i \tilde{p} \tilde{x}}, \\
& \Pi(\tilde{x})=(2 \pi)^{-3 / 2} \int d^{2} \tilde{p} d \tilde{p}_{-} \sqrt{\frac{\omega_{p}}{2}}\left[a(\tilde{p})-a^{+}(-\tilde{p})\right] e^{-i \tilde{p} \tilde{x}},
\end{aligned}
$$


где $\tilde{p} \tilde{x} \equiv p_{\perp} x^{\perp}+\tilde{p}_{-} \tilde{x}^{-}, \omega_{p}=\left(\tilde{p}_{-}^{2}+\eta^{2}\left(p_{\perp}^{2}+m^{2}\right)\right)^{1 / 2}$. Эти операторы удовлетворяют при $\tilde{x}^{+}=0$ перестановочным соотношениям

$$
\left[a(\tilde{p}), a^{+}(\tilde{q})\right]=\delta^{3}(\tilde{p}-\tilde{q}), \quad[a(\tilde{p}), a(\tilde{q})]=\left[a^{+}(\tilde{p}), a^{+}(\tilde{q})\right]=0 .
$$

Свободную часть гамильтониана можно записать в виде

$$
H_{0}=\int d^{2} p_{\perp} d \tilde{p}_{-} \frac{\omega_{p}-\tilde{p}_{-}}{\eta^{2}} a^{+}(\tilde{p}) a(\tilde{p}) .
$$

Если ограничить моды поля по $\tilde{p}_{-}$путем обрезания $\left|\tilde{p}_{-}\right| \geqslant \varepsilon$, а также предположить нормально упорядоченную форму гамильтониана взаимодействия (что сделано в $(20))$, то, как легко видеть, в пределе $\eta \rightarrow 0$ при условии конечности энергии мы приходим к канонической формулировке на СФ на подпространстве $\left|f_{0}\right\rangle$ фоковского пространства, определяемом условиями

$$
a(\tilde{p})\left|f_{0}\right\rangle=0, \quad \tilde{p}_{-} \leqslant-\varepsilon .
$$

Это подпространство в пределе становится фоковским пространством на СФ

Для того чтобы приближенно учесть вакуумные эффекты, примем во внимание также окрестность $\tilde{p}_{-}=0$, включая в рассмотрение моды поля с $\left|\tilde{p}_{-}\right| \leqslant \Lambda \eta$, где $\Lambda$ - некоторая величина, имеющая размерность импульса. При $\eta \rightarrow 0$ моды поля в интервале $\Lambda \eta<\left|\tilde{p}_{-}\right|<\varepsilon$ оказываются отброшенными. Мы предполагаем, что это искажение теории можно считать несущественным в пределе $\eta \rightarrow 0$, если после перехода к этому пределу положить $\Lambda \rightarrow \infty$ (после соответствующей ультрафиолетовой перенормировки теории).

Проведем предельный переход в рамках теории возмущений по малому параметру $\eta$ для решений уравнения Шредингера с гамильтонианом (17)

$$
\widetilde{H}(\eta)|f(\eta)\rangle=\widetilde{E}(\eta)|f(\eta)\rangle,
$$

описывающих состояния с конечной энергией (и массой). С этой целью разложим гамильтониан по степеням $\eta$ :

$$
\widetilde{H}(\eta)=\frac{1}{\eta^{2}} H^{(0)}+\frac{1}{\eta} H^{(1)}+H^{(2)}+\cdots
$$

Члены этого разложения получаются подстановкой в гамильтониан разложения поля на части, соответствующие введенному выше разделению мод

$$
\varphi(\tilde{x}) \approx \check{\varphi}_{\varepsilon}(\tilde{x})+\varphi_{\Lambda \eta}(\tilde{x}),
$$

где $\check{\varphi}_{\varepsilon}(\tilde{x})$ включает только моды с $\left|\tilde{p}_{-}\right| \geqslant \varepsilon$, а $\varphi_{\Lambda \eta}(\tilde{x})-$ только моды с $\left|\tilde{p}_{-}\right| \leqslant \Lambda \eta$. Аналогичное разложение предполагается для П $(\tilde{x})$.

Свободная (квадратичная по полям) часть гамильтониана (23) может быть записана в виде

$$
H_{0}(\Pi, \varphi) \approx H_{0}\left(\check{\Pi}_{\varepsilon}, \check{\varphi}_{\varepsilon}\right)+H_{0}\left(\Pi_{\Lambda \eta}, \varphi_{\Lambda \eta}\right),
$$

а взаимодействие $H_{\mathrm{I}}$ - в виде

$$
H_{\mathrm{I}}=\lambda \int d^{2} x^{\perp} d \tilde{x}^{-} \varphi^{4}(\tilde{x}) \approx H_{\mathrm{I}}\left(\check{\varphi}_{\varepsilon}\right)+H_{\mathrm{I}}\left(\varphi_{\Lambda \eta}\right)+\lambda \int d^{2} x^{\perp} d \tilde{x}^{-}\left(6 \varphi_{\Lambda \eta}^{2} \check{\varphi}_{\varepsilon}^{2}+4 \varphi_{\Lambda \eta} \check{\varphi}_{\varepsilon}^{3}\right) .
$$


Под интегралом отброшен член $\varphi_{\Lambda \eta}^{3} \check{\varphi}_{\varepsilon}$, так как в интересующем нас пределе $\eta \rightarrow 0$ величина $\varphi_{\Lambda \eta}^{3}$ становится постоянной, а интеграл по $\tilde{x}^{-}$от $\check{\varphi}_{\varepsilon}$ равен нулю.

Используя представление для полей через операторы рождения и уничтожения, получаем

$$
\begin{aligned}
H_{0}\left(\check{\Pi}_{\varepsilon}, \check{\varphi}_{\varepsilon}\right)= & \frac{1}{\eta^{2}} \int d^{2} p_{\perp} \int_{\left|\tilde{p}_{-}\right| \geqslant \varepsilon} d \tilde{p}_{-}\left[\sqrt{\tilde{p}_{-}^{2}+\eta^{2}\left(p_{\perp}^{2}+m^{2}\right)}-\tilde{p}_{-}\right] a^{+}(\tilde{p}) a(\tilde{p})= \\
= & \frac{2}{\eta^{2}} \int d^{2} p_{\perp} \int_{p_{-} \leqslant-\varepsilon} d p_{-}\left|p_{-}\right| a^{+}(p) a(p)+ \\
& +\int d^{2} p_{\perp} \int_{p_{-} \geqslant \varepsilon} d p_{-} \frac{m^{2}+p_{\perp}^{2}}{2 p_{-}} a^{+}(p) a(p)+O\left(\eta^{2}\right) .
\end{aligned}
$$

С другой стороны, поля $\varphi_{\Lambda \eta}, \Pi_{\Lambda \eta}$ можно представить в “быстродвижущейся" лоренцевой системе координат следующим образом:

$$
\begin{aligned}
\left.\varphi_{\Lambda \eta}(\tilde{x})\right|_{\tilde{x}^{+}=0} & =\left.\varphi_{\Lambda}\left(-x^{\prime 3}, x^{\perp}\right)\right|_{x^{\prime 0}=0}, \\
\left.\Pi_{\Lambda \eta}(\tilde{x})\right|_{\tilde{x}^{+}=0} & =\left.\eta \Pi_{\Lambda}\left(-x^{\prime 3}, x^{\perp}\right)\right|_{x^{\prime 0}=0}
\end{aligned}
$$

где $\Lambda$ - обрезание по импульсу $p_{3}^{\prime},\left|p_{3}^{\prime}\right| \leqslant \Lambda$. Отсюда легко вывести, что

$$
\widetilde{H}\left(\Pi_{\Lambda \eta}, \varphi_{\Lambda \eta}\right)=H_{0}\left(\Pi_{\Lambda \eta}, \varphi_{\Lambda \eta}\right)+H_{\mathrm{I}}\left(\varphi_{\Lambda \eta}\right)=\frac{1}{\eta}\left(P_{0}+P_{3}\right)_{\Lambda, x^{\prime 0}=0}
$$

с точностью до возможного добавления константы, делающей квантовый оператор в правой части равенства неотрицательным, а его минимум равным нулю.

В результате получаем

$$
\begin{gathered}
H^{(0)}=2 \int d^{2} p_{\perp} \int_{p_{-} \leqslant-\varepsilon} d p_{-}\left|p_{-}\right| a^{+}(p) a(p), \\
H^{(1)}=\left(P_{0}+P_{3}\right)_{\Lambda, x^{\prime 0}=0}, \\
H^{(2)}=\int d^{2} p_{\perp} \int_{p_{-} \geqslant \varepsilon} d p_{-} \frac{m^{2}+p_{\perp}^{2}}{2 p_{-}} a^{+}(p) a(p)+ \\
+\lambda \int d^{2} x^{\perp} d x^{-}\left(\left.6 \varphi_{\Lambda}^{2}(x)\right|_{x^{\prime 0}=0} \check{\varphi}_{\varepsilon}^{2}(x)+\left.4 \varphi_{\Lambda}(x)\right|_{x^{\prime 0}=0} \check{\varphi}_{\varepsilon}^{3}(x)+\check{\varphi}_{\varepsilon}^{4}(x)\right) .
\end{gathered}
$$

Теперь построим теорию возмущений для уравнения Шредингера (22), вводя разложения по степеням $\eta$ :

$$
\begin{gathered}
|f(\eta)\rangle=\left|f^{(0)}\right\rangle+\eta\left|f^{(1)}\right\rangle+\eta^{2}\left|f^{(2)}\right\rangle+\cdots, \\
\widetilde{E}(\eta)=E+O(\eta),
\end{gathered}
$$

где учтено требование конечности энергии $E(\eta)$ в пределе $\eta \rightarrow 0$. В низшем порядке по $\eta$ имеем

$$
H^{(0)}\left|f^{(0)}\right\rangle=0
$$

Отсюда следует, что

$$
a(p)\left|f^{(0)}\right\rangle=0, \quad p_{-} \leqslant-\varepsilon .
$$


Далее,

$$
H^{(1)}\left|f^{(0)}\right\rangle+H^{(0)}\left|f^{(1)}\right\rangle=0 .
$$

Отсюда в силу равенства (34)

$$
\left\langle f^{(0)}\left|H^{(1)}\right| f^{(0)}\right\rangle=0
$$

или, что эквивалентно,

$$
\left\langle f^{(0)}\left|\left(P_{0}+P_{3}\right)_{\Lambda, x^{\prime 0}=0}\right| f^{(0)}\right\rangle=0 .
$$

Таким образом, мы приходим к выводу, что зависимость состояния $\left|f^{(0)}\right\rangle$ от мод полей $\varphi_{\Lambda \eta}, \Pi_{\Lambda \eta}$ должна отвечать минимуму оператора $\left(P_{0}+P_{3}\right)_{\Lambda, x^{\prime 0}=0}$. Вводя для этой зависимости обозначение $\left|\operatorname{vac}_{\Lambda}\right\rangle$, получаем общий вид базиса для состояний $\left|f^{(0)}\right\rangle$ :

$$
\left\{\left|f^{(0)}\right\rangle\right\}=\left\{\prod_{n, p_{n} \geqslant \varepsilon} a^{+}\left(p_{n}\right)|0\rangle\right\} \otimes\left|\operatorname{vac}_{\Lambda}\right\rangle_{x^{\prime 0}=0} .
$$

В следующем порядке по $\eta$ имеем

$$
H^{(2)}\left|f^{(0)}\right\rangle+H^{(1)}\left|f^{(1)}\right\rangle+H^{(2)}\left|f^{(0)}\right\rangle=E\left|f^{(0)}\right\rangle .
$$

Отсюда следует, что значения $E$ определяются набором собственных значений оператора

$$
\begin{aligned}
& \mathcal{P}_{0} H^{(2)} \mathcal{P}_{0}=H_{\mathrm{LF}}=\int d^{2} p_{\perp} \int_{p_{-} \geqslant \varepsilon} d p_{-} \frac{m^{2}+p_{\perp}^{2}}{2 p_{-}} a^{+}(p) a(p)+ \\
& +\lambda \int d^{2} x^{\perp} d x^{-}\left(\check{\varphi}_{\varepsilon}^{4}(x)+4\left\langle\operatorname{vac}_{\Lambda}\left|\varphi_{\Lambda}\right| \operatorname{vac}_{\Lambda}\right\rangle \check{\varphi}_{\varepsilon}^{3}(x)+6\left\langle\operatorname{vac}_{\Lambda}\left|\varphi_{\Lambda}^{2}\right| \operatorname{vac}_{\Lambda}\right\rangle \check{\varphi}_{\varepsilon}^{2}(x)\right) \underset{\Lambda \rightarrow \infty}{\longrightarrow} \\
& \underset{\Lambda \rightarrow \infty}{\longrightarrow} H_{\mathrm{LF}}^{\mathrm{can}}\left(\check{\varphi}_{\varepsilon}\right)+\lambda \int d^{2} x^{\perp} d x^{-}\left(4\langle\varphi\rangle_{\operatorname{vac}} \check{\varphi}_{\varepsilon}^{3}(x)+6\left\langle\varphi^{2}\right\rangle_{\operatorname{vac}} \check{\varphi}_{\varepsilon}^{2}(x)\right),
\end{aligned}
$$

где $\mathcal{P}_{0}$ обозначает проектор на подпространство состояний $\left|f^{(0)}\right\rangle$, а $\left\langle\varphi^{n}\right\rangle_{\text {vac }}$ - результат усреднения вкладов соответствующих мод полей по $\left|\operatorname{vac}_{\Lambda}\right\rangle$. В рамках описанного

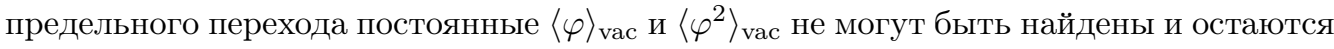
свободными параметрами.

Последнее соотношение определяет искомое приближенное выражение для гамильтониана на СФ и его отличие от выражения $H_{\mathrm{LF}}^{\mathrm{can}}$, получаемого непосредственным квантованием на СФ

Отметим, что данный результат для гамильтониана на СФ согласуется с ковариантной теорией возмущений по константе взаимодействия во всех порядках [3].

Однако описанный выше способ построения гамильтониана на СФ сталкивается с трудностями для калибровочных теорий, где роль динамических переменных играют калибровочно-неинвариантные поля. Трудно ввести регуляризации, сохраняющие лоренцеву и калибровочную симметрии. Кроме того, предложенная выше процедура отделения одной части фурье-мод этих полей от другой в пренебрежении промежуточными модами тоже нарушает эти симметрии. Наличие обусловленных 
калибровочной симметрией связей между каноническими переменными усложняет применение вышеописанной процедуры к исследованию предельного перехода на СФ, поскольку эти связи нелинейны по полям, и результат может зависеть от того, какие из переменных выбраны независимыми (и подлежат разделению на части после перехода к фурье-модам).

\section{3. КАЛИБРОВОЧНАЯ ТЕОРИЯ НА РЕШЕТКЕ}

Ограничимся далее описанием калибровочно-инвариантного подхода к построению гамильтониана в координатах $\tilde{x}$, близких к координатам СФ, в качестве предварительного этапа на пути формулировки и исследования предельного перехода на СФ для теории калибровочного поля. Этот подход использует пространственновременну́ю решетку [12] в указанных координатах и аналогичен методу работы [13].

Введем решетку по координатам $\tilde{x}^{+}, \tilde{x}^{-}, \tilde{x}^{\perp}$ с параметрами $a_{+}, a_{-}, a_{\perp}$, обозначающими расстояния между узлами решетки вдоль соответствующих координат в масштабе этих координат. Калибровочные поля описываются унитарными $(N \times N)$ матрицами $U_{\mu}(\tilde{x})$, принадлежащими группе $S U(N)$. Эти матрицы сопоставляются ребрам решетки, как показано на рисунке:
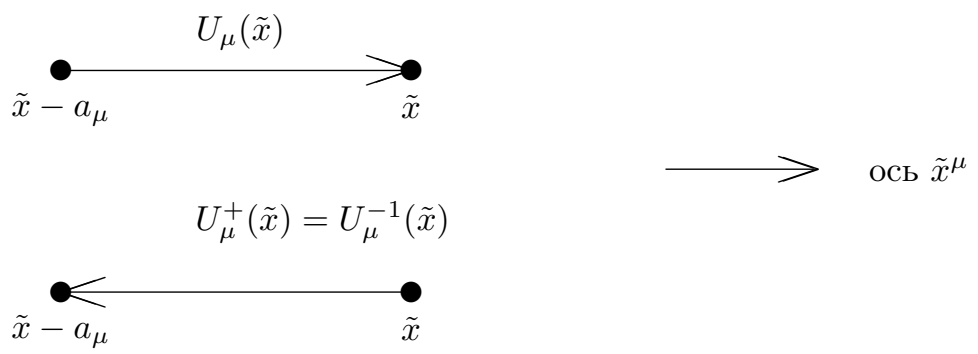

При калибровочных преобразованиях $\Omega(\tilde{x})$, отвечающих фундаментальному представлению группы $S U(N)$, матрицы преобразуются следующим образом:

$$
U_{\mu}(\tilde{x}) \stackrel{\Omega}{\longrightarrow} \Omega(\tilde{x}) U_{\mu}(\tilde{x}) \Omega^{-1}\left(\tilde{x}-a_{\mu}\right) .
$$

Формальный переход к теории в непрерывном пространстве с калибровочными полями $\tilde{A}_{\mu}(\tilde{x})=\tilde{A}_{\mu}^{a}(\tilde{x}) \lambda^{a} / 2$, где $\lambda^{a} / 2$ - аналог матриц Гелл-Мана для группы $S U(N)$, соответствует представлению

$$
U_{\mu}(\tilde{x}) \approx e^{i a_{\mu} \tilde{A}_{\mu}(\tilde{x})} \approx 1+i a_{\mu} \tilde{A}_{\mu}(\tilde{x})+\cdots
$$

при условии $a_{\mu} \rightarrow 0$, где $\tilde{A}_{\mu}-$ векторы, отнесенные к координатам $\tilde{x}$.

Определим также "плакетные" переменные $U_{\mu \nu}(x)$ :

$$
\begin{gathered}
U_{\mu \nu}(\tilde{x})=U_{\mu}(\tilde{x}) U_{\nu}\left(\tilde{x}-a_{\mu}\right) U_{\mu}^{-1}\left(\tilde{x}-a_{\nu}\right) U_{\nu}^{-1}(\tilde{x}), \\
U_{\mu \nu}(\tilde{x})=U_{\nu \mu}^{+}(\tilde{x})=U_{\nu \mu}^{-1}(\tilde{x}) .
\end{gathered}
$$

При калибровочных преобразованиях имеем

$$
U_{\mu \nu}(\tilde{x}) \stackrel{\Omega}{\longrightarrow} \Omega(\tilde{x}) U_{\mu \nu}(\tilde{x}) \Omega^{-1}(\tilde{x}),
$$

4 Теоретическая и математическая физика, т. 148, № 1,2006 г. 
так что величина $\operatorname{tr} U_{\mu \nu}(x)$ калибровочно инвариантна.

Аналогом формулы (43) для плакетных переменных служит следующее выражение:

$$
U_{\mu \nu}(\tilde{x}) \approx e^{i a_{\mu} a_{\nu} \widetilde{F}_{\mu \nu}(\tilde{x})} \approx 1+i a_{\mu} a_{\nu} \widetilde{F}_{\mu \nu}(\tilde{x})-\frac{1}{2} a_{\mu}^{2} a_{\nu}^{2} \widetilde{F}_{\mu \nu}^{2}(\tilde{x})+\cdots
$$

где $\widetilde{F}_{\mu \nu}=\partial_{\mu} \tilde{A}_{\nu}-\partial_{n} \tilde{A}_{\mu}-i\left[\tilde{A}_{\mu}, \tilde{A}_{\nu}\right]$.

Формулы (43),(46) позволяют представить выражение, аппроксимирующее лагранжево действие непрерывной теории, в терминах решеточных переменных, используя следующие предписания:

$$
\begin{gathered}
a_{\mu} a_{\nu} \widetilde{F}_{\mu \nu}(\tilde{x}) \longrightarrow \frac{1}{2 i}\left(U_{\mu \nu}(\tilde{x})-U_{\mu \nu}^{+}(\tilde{x})\right) \equiv \operatorname{Im} U_{\mu \nu}(\tilde{x}), \\
a_{\mu}^{2} a_{\nu}^{2} \operatorname{tr} \widetilde{F}_{\mu \nu}^{2}(\tilde{x}) \longrightarrow \operatorname{tr}\left(2-U_{\mu \nu}(\tilde{x})-U_{\mu \nu}^{+}(\tilde{x})\right) \equiv 2 \operatorname{tr}\left(1-\operatorname{Re} U_{\mu \nu}(\tilde{x})\right) .
\end{gathered}
$$

В близких к СФ координатах (13) имеем в непрерывном случае

$$
\begin{gathered}
S=\int d^{4} \tilde{x} L(\tilde{x}), \\
L(\tilde{x})=-\frac{1}{4 g^{2}} \widetilde{F}_{\mu \nu}^{a}(\tilde{x}) \widetilde{F}_{\rho \lambda}^{a}(\tilde{x}) \tilde{g}^{\rho \mu}(\tilde{x}) \tilde{g}^{\lambda \nu}(\tilde{x})= \\
=\frac{1}{2 g^{2}} \widetilde{F}_{+-}^{a}(\tilde{x}) \widetilde{F}_{+-}^{a}(\tilde{x})+\sum_{k=1,2} \frac{1}{g^{2}}\left(\frac{\eta^{2}}{2} \widetilde{F}_{+k}^{a}(\tilde{x}) \widetilde{F}_{+k}^{a}(\tilde{x})+\widetilde{F}_{+k}^{a}(\tilde{x}) \widetilde{F}_{-k}^{a}(\tilde{x})\right)+ \\
+\frac{1}{2 g^{2}} \widetilde{F}_{12}^{a}(\tilde{x}) \widetilde{F}_{12}^{a}(\tilde{x}) .
\end{gathered}
$$

Для действия на решетке получаем

$$
\begin{aligned}
S_{\text {lat }}= & \frac{2 a_{+} a_{-} a_{\perp}^{2}}{g^{2}} \sum_{x} \operatorname{tr}\left(\frac{\operatorname{Re}\left(1-U_{+-}(\tilde{x})\right)}{a_{+}^{2} a_{-}^{2}}+\sum_{k=1,2} \frac{\eta^{2} \operatorname{Re}\left(1-U_{+k}(\tilde{x})\right)}{a_{+}^{2} a_{\perp}^{2}}+\right. \\
& \left.+\sum_{k=1,2} \frac{\operatorname{Im} U_{+k}(\tilde{x}) \operatorname{Im} U_{-k}(\tilde{x})}{a_{+} a_{-} a_{\perp}^{2}}-\frac{\operatorname{Re}\left(1-U_{12}(\tilde{x})\right)}{a_{\perp}^{4}}\right) .
\end{aligned}
$$

Чтобы вывести аналог гамильтониана, рассмотрим решеточное представление функционального интеграла [12] для матричных элементов оператора эволюции [13] (аналогичное рассмотрение в непрерывном пространстве см. в книге [14]). В качестве параметра эволюции используется координата $\tilde{x}^{+}$(в последующем рассматривается предел $\left.a_{+} \rightarrow 0\right)$, а также вводится калибровочное условие

$$
U_{+}(\tilde{x})=1 .
$$

При таком условии легко найти [13] вид квантового оператора эволюции $\widetilde{T}_{+}$, связывающего базисные состояния (собственные значения, отвечающие этим состояниям, служат переменными интегрирования в функциональном интеграле) в моменты $\tilde{x}^{+}$, отличающиеся на величину $a_{+}$. Оператор $\widetilde{T}_{+}$, в свою очередь, связан с гамильтонианом $\widetilde{H}$ в координатах $\tilde{x}$ в пределе $a_{+} \rightarrow 0$ :

$$
\widetilde{T}_{+}=\exp \left(-i a_{+} \widetilde{H}+O\left(a_{+}^{2}\right)\right) .
$$


По аналогии с работой [13] введем используемый при определении функционального интеграла базис состояний

$$
\begin{gathered}
|U\rangle=\prod_{i, \tilde{x}}\left|U_{i}(\tilde{x})\right\rangle, \quad i=-, 1,2, \\
\widehat{U}_{i}(\tilde{x})|U\rangle=U_{i}(\tilde{x})|U\rangle, \quad\left\langle U^{\prime} \mid U\right\rangle=\delta\left(U^{\prime}, U\right), \quad 1 \equiv \int d U|U\rangle\langle U|,
\end{gathered}
$$

где $d U$ - инвариантная мера на группе матриц $U$.

Определим унитарные операторы $\widehat{R}_{i, \tilde{x}}\left(g_{i}\right)$ по аналогии с операцией “сдвига" на группе матриц $U$ :

$$
\widehat{R}_{i, \tilde{x}}\left(g_{i}\right)\left|U_{i}(\tilde{x})\right\rangle=\left|g_{i} U_{i}(\tilde{x})\right\rangle,
$$

где $g_{i}$ - произвольная $S U(N)$-матрица размера $N \times N$.

Легко проверить, что оператор $\widetilde{T}_{+}$можно записать следующим образом [13]:

$$
\begin{aligned}
\widetilde{T}_{+}=\prod_{k} \prod_{\tilde{x}^{-}, \tilde{x}^{\perp}} \int d g_{-} d g_{\perp} \widehat{R}_{-, \tilde{x}}\left(g_{-}\right) \widehat{R}_{k, \tilde{x}}\left(g_{k}\right) \times \\
\quad \times \exp \left[-\frac{2 i}{g^{2}} \operatorname{tr}\left(\frac{g_{-}+g_{-}^{+}}{2 a_{+} a_{-}}\right)-\frac{2 i \eta^{2} a_{-}}{g^{2} a_{+}} \operatorname{tr}\left(\frac{g_{k}+g_{k}^{+}}{2}\right)-\right. \\
\left.\quad-\frac{2 i}{g^{2}} \operatorname{tr}\left(\frac{g_{k}-g_{k}^{+}}{2 i} \operatorname{Im} U_{-k}(\tilde{x})+\frac{2 i a_{+} a_{-}}{g^{2} a_{\perp}^{2}} \operatorname{Re} U_{12}(\tilde{x})\right)\right] .
\end{aligned}
$$

Параметризуем матрицы $g_{i}$ с помощью вещественных параметров $\theta_{i}^{a}$ :

$$
g_{i}=\exp \left(i \theta_{i}^{a} \frac{\lambda^{a}}{2}\right)
$$

Для операторов $\widehat{R}_{i, \tilde{x}}\left(g_{i}\right)$ получаем

$$
\widehat{R}_{i, \tilde{x}}\left(g_{i}\right)=e^{i \theta_{i}^{a} \widehat{\Pi}_{i}^{a}(\tilde{x})},
$$

где операторы $\widehat{\Pi}_{i}^{a}(\tilde{x})$ реализуют представление матриц $\lambda^{a} / 2$ и подчиняются коммутационным соотношениям

$$
\left[\widehat{\Pi}_{i}^{a}(\tilde{x}), \widehat{\Pi}_{i}^{b}(\tilde{x})\right]=i f^{a b c} \widehat{\Pi}_{i}^{c}(\tilde{x}),
$$

$f^{a b c}$ - структурные константы группы $S U(N)$ :

$$
\left[\frac{\lambda^{a}}{2}, \frac{\lambda^{b}}{2}\right]=i f \frac{f^{a b c}}{2} .
$$

В пределе $a_{+} \rightarrow 0$ интегралы по $g_{i}$ в выражении для $\widetilde{T}_{+}$можно вычислить в полной аналогии с работой [13]. В результате приходим к следующему выражению для гамильтониана:

$$
\begin{aligned}
\widetilde{H}(\eta)= & \sum_{\tilde{x}^{-}, x^{\perp}}\left[\frac{g^{2} a_{-}}{2 a_{\perp}^{2}} \widehat{\Pi}_{-}^{a}(\tilde{x}) \widehat{\Pi}_{-}^{a}(\tilde{x})+\right. \\
& \left.\quad+\frac{g^{2}}{2 \eta^{2} a_{-}} \sum_{k=1,2}\left(\widehat{\Pi}_{k}^{a}(\tilde{x})-\frac{1}{g^{2}} \operatorname{tr}\left(\lambda^{a} \operatorname{Im} U_{-, k}(\tilde{x})\right)\right)^{2}-\frac{2 a_{-}}{g^{2} a_{\perp}^{2}} \operatorname{tr} \operatorname{Re} U_{12}(\tilde{x})\right] .
\end{aligned}
$$


Вследствие формул (53), (56) имеем соотношение

$$
e^{-i \theta_{i}^{a} \widehat{\Pi}_{i}^{a}} \widehat{U}_{i} e^{i \theta_{i}^{a} \widehat{\Pi}_{i}^{a}}=e^{i \theta_{i}^{a} \lambda^{a} / 2} \widehat{U}_{i},
$$

из которого можно получить для операторов $\widehat{\Pi}_{i}^{a}(\tilde{x})$ и $\widehat{U}_{i}(\tilde{x})$ следующие перестановочные соотношения:

$$
\left[\widehat{\Pi}_{i}^{a}(\tilde{x}), \widehat{U}_{i}(\tilde{x})\right]=-\frac{\lambda^{a}}{2} \widehat{U}_{i}(\tilde{x}) .
$$

Легко проверить, что форма этих соотношений остается неизменной при калибровочном преобразовании переменных, если для $\widehat{\Pi}_{i}^{a}(x)$ выполняется следующий закон преобразования:

$$
\widehat{\Pi}_{i}(\tilde{x}) \stackrel{\Omega}{\longrightarrow} \Omega(\tilde{x}) \widehat{\Pi}_{i}(\tilde{x}) \Omega^{-1}(\tilde{x}),
$$

где $\widehat{\Pi}_{i}(\tilde{x})=\left(\lambda^{a} / 2\right) \widehat{\Pi}_{i}^{a}(\tilde{x})$. Для получения данного результата достаточно заметить, что матрицы $\lambda^{a}$ играют роль векторов в присоединенном представлении группы $S U(N)$.

Калибровочное условие (50) сохраняет симметрию относительно калибровочных преобразований, не зависящих от $\tilde{x}^{+}$. Генераторы этих преобразований соответствуют каноническим связям, которые в квантовой теории рассматриваются как условия, определяющие физическое подпространство состояний [15]. Векторы этого подпространства состояний, описываемые функционалами от полей, должны быть калибровочно инвариантны. На пространственной решетке конечных размеров указанные состояния соответствуют функциям от следов произведений матриц $U_{i}(\tilde{x})$ вдоль возможных замкнутых контуров, проводимых по ребрам решетки. Гамильтониан $\widetilde{H}(\eta)$ надо рассматривать только на таких состояниях. Физический вакуум должен отвечать минимуму гамильтониана. Кроме того, вакуумное состояние предполагается инвариантным относительно сдвигов вдоль координат $\tilde{x}^{-}, \tilde{x}^{\perp}$. Аналогично при нахождении спектра связанных состояний можно фиксировать подпространство состояний, инвариантных относительно сдвигов вдоль $\tilde{x}^{\perp}$ и приобретающих определенный фазовый множитель при сдвиге вдоль $\tilde{x}^{-}$(это соответствует использованию уравнений (3) в непрерывной теории).

Очевидно, что точное решение данной задачи столь же сложно, как и в обычных лоренцевых координатах с гамильтонианом при фиксированном времени $x^{0}$. Переход к координатам $\tilde{x}$, близким к координатам СФ и содержащим малый параметр $\eta$, позволяет использовать малость $\eta$, если сделать простые предположения относительно возможного приближения к точному решению, как это было продемонстрировано выше для теории скалярного поля в непрерывном пространстве. Можно, например, развить теорию возмущений по параметру $\eta$ для уравнения на собственные значения гамильтониана $\widetilde{H}(\eta)$, фиксируя значения параметров решетки и ее пространственные размеры (накладывая периодические граничные условия для полей по пространственным координатам). Исследование такой теории возмущений в непрерывном пространстве (с фиксированным параметром обрезания $\left|x^{-}\right| \leqslant L$ и периодическими граничными условиями по координате $x^{-}$) для двумерной квантовой электродинамики [5], [16] показало, что с помощью феноменологически подобранной модификации членов гамильтониана, содержащих нулевые моды поля, удается достичь адекватного учета вакуумных эффектов. Это позволяет надеяться, что аналогичный результат можно получить и в рамках решеточного подхода. 
С другой стороны, с помощью теории возмущений по $\eta$ можно строить вакуумное состояние на решетке, описываемое функционалом от калибровочных полей, ограничиваясь низшими порядками по $\eta$, а затем использовать этот функционал для приближенного вычисления квантовых корреляторов, сохраняя конечное, хотя и малое, значение параметра $\eta$. Такой подход может быть полезен при анализе рассеяния адронов при высоких энергиях.

Благодарности. Авторы благодарят региональное бюро ЮНЕСКО по научному сотрудничеству в Европе за поддержку Международной школы физики им. В. А. Фока. Работа С. Пастона и Е. Прохватилова поддержана РФФИ (грант № 05-02-17477), а также Федеральным агентством по образованию РФ (грант № РНП.2.1.1.1112).

\section{Список литературы}

[1] P. A. M. Dirac, Rev. Mod. Phys., 21 (1949), 392.

[2] P. M. Stevenson, Phys. Rev. D, 32 (1985), 1389.

[3] С. А. Пастон, В. А. Франке, ТМФ, 112:3 (1997), 399; hep-th/9901110.

[4] С. А. Пастон, Е.В. Прохватилов, В.А. Франке, ТМФ, 120:3 (1999), 417; hep-th/0002062; 131:1 (2002), 84; hep-th/0302016.

[5] E. V. Prokhvatilov, H. W. L. Naus, H.-J. Pirner, Phys. Rev. D, 51 (1995), 2933; hep-ph/9406275.

[6] Е. В. Прохватилов, В. А. Франке, ЯФ, 59:11 (1996), 2105.

[7] S. Dalley, G. McCartor, Annals Phys., 321 (2006), 402; hep-ph/0406287.

[8] V. A. Franke, Yu. V. Novozhilov, E. V. Prokhvatilov, Lett. Math. Phys., 5:3 (1981), 239; № $5,437$.

[9] А. М. Анненкова, Е. В. Прохватилов, В. А. Франке, Вестник ЛГУ, 1985, № 4, 80; С. А. Пастон, Е. В. Прохватилов, В.А. Франке, ЯФ, 68:2 (2005), 1; hep-th/0501186; S. J. Brodsky, H.-C. Pauli, S. S. Pinsky, Phys. Rep., 301 (1997), 299; hep-ph/9705477.

[10] А. М. Анненкова, Е. В. Прохватилов, В. А. Франке, ЯФ, 56:6 (1993), 179.

[11] K. G. Wilson, T. Walhout, A. Harindranath, W. M. Zhang, R. J. Perry, S. Glazek, Phys. Rev. D, 49 (1994), 6720; hep-th/9401153; S. J. Brodsky, V. A. Franke, J. R. Hiller, G. McCartor, S. A. Paston, E. V. Prokhvatilov, Nucl. Phys. B, 703 (2004), 333; hep-ph/0406325.

[12] K. Wilson, Phys. Rev. D, 10 (1974), 2445.

[13] M. Creutz, Phys. Rev. D, 15 (1977), 1128.

[14] А.А. Славнов, Л. Д. Фаддеев, Введение в квантовую теорию калибровочных полей, Наука, М., 1988.

[15] П.А. М. Дирак, Лекиии по квантовой механике, Мир, М., 1968.

[16] Е. В. Прохватилов, В. А. Франке, ЯФ, 47:3 (1988), 882; 49:4 (1989), 1109; А. Б. Былев, Е. В. Прохватилов, В.А. Франке, Вестник ЛГУ, Сер. 4, Вып. 2, 1989, № 11, 66. 